\title{
Peroxisome proliferator-activated receptor delta regulates lipid droplet formation and transport in goat mammary epithelial cells
}

\author{
H. B. Shi, ${ }^{\dagger} †$ C. H. Zhang, $\ddagger$ Z. A. Xu, ${ }^{*}$ G. G. Lou, ${ }^{*}$ J. X. Liu, $\dagger$ J. Luo, $\ddagger^{1}$ and J. J. Loor $\S^{1}$ \\ *Zhejiang Provincial Key Laboratory of Silkworm Bioreactor and Biomedicine, College of Life Sciences, Zhejiang Sci-Tech University, Hangzhou, \\ 310018, P. R. China \\ †College of Animal Sciences, Zhejiang University, Hangzhou 310058, P. R. China \\ ¥College of Animal Science and Technology, Northwest A\&F University, Yangling, Shaanxi, 712100, P. R. China \\ $\S$ Mammalian NutriPhysioGenomics, Department of Animal Sciences and Division of Nutritional Sciences, University of Illinois, Urbana 61801
}

\section{ABSTRACT}

Even though recent evidence in goat mammary epithelial cells (GMEC) suggest a role of peroxisome proliferator-activated receptor delta (PPARD) in regulating lipid homeostasis, its role is not fully understood. Our hypothesis was that PPARD regulates lipid transport processes in GMEC and, thus, plays a crucial role in regulating fat formation. The PPARD was overexpressed using an adenovirus system (AdPPARD) with recombinant green fluorescent protein (Ad-GFP) as the control. Results revealed that overexpression of PPARD markedly upregulated the mRNA abundance of PPARD. Compared with the control (AdGFP+dimethyl sulfoxide), overexpression of PPARD alone had no effect on mRNA expression of CD36, $S C D 1, F A B P 4, A C S L 1$, and ADRP. The cultures overexpressing PPARD with the PPARD ligand GW0742 (GW) upregulated the expression of CD36, FABP3, $F A B P 4, A C S L 1$, and $A D R P$. Overexpression of PPARD in GMEC plus GW increased the concentration of 16:1 and 18:1-trans and was associated with upregulation of $S C D 1$. Compared with the control (Ad-GFP+dimethyl sulfoxide), the decrease of triacylglycerol concentration coupled with upregulation of genes related to lipid droplet secretion (e.g., $A D R P$ and ACSL1) induced by $P P A R D$ overexpression suggests a role in lipid droplet (LD) secretion. Luciferase assay revealed that GW increased the ADRP promoter activity in a dosedependent manner. Knockdown of PPARD impaired the increase of ADRP promoter activity induced by GW, whereas GW enhanced the activity of ADRP promoter in GMEC overexpressing PPARD. Data with the $A D R P 5^{\prime}$-flanking truncated luciferase reporter suggest a core region $(-1,444$ to $-990 \mathrm{bp})$ response element

\footnotetext{
Received July 21, 2017.

Accepted November 27, 2017.

${ }^{1}$ Corresponding authors: luojun@nwsuaf.edu.cn and jloor@illinois. edu
}

for the induction of GW. This core region contains a known PPARG response element (PPRE) at $-1,003$ to -990 bp. When the PPRE was mutated, the overexpression of PPARD had no effect on ADRP promoter activity. Collectively, these results reveal a novel role for PPARD in lipid homeostasis via promoting fatty acid transport and LD formation through a mechanism of direct binding to the promoter of key genes. Hence, PPARD activity may contribute to fatty acid transport and LD formation during lactation.

Key words: perilipin, promoter, peroxisome proliferator-activated receptor response element

\section{INTRODUCTION}

Transcription factors play important roles regulating fatty acid metabolism in ruminant mammary tissue (Harvatine et al., 2009; Oppi-Williams et al., 2013). Peroxisome proliferator-activated receptors (PPAR) are nuclear proteins that regulate the synthesis of lipid or lipid oxidation upon activation by endogenous or synthetic agonists (Bionaz et al., 2013). In the ruminant, the role of PPAR $\alpha$ (PPARA) (Bionaz et al., 2013) and PPAR $\gamma$ (PPARG; Shi et al., 2013b, 2014b) in controlling lipid metabolism in mammary tissue has been well studied. Despite data indicating that its function seems to overlap with PPARA in terms of fatty acid (FA) oxidation (Giordano Attianese and Desvergne, 2015), compared with PPARG and PPARA, the biologic role of PPAR delta (PPARD) remains unclear. The finding in mice that PPARD activates an array of genes required for FA degradation and uncoupling suggested an opposite role of PPARD compared with PPARG (Wang et al., 2003). Thus, exploring and understanding the regulatory role of PPARD in lipid metabolism in mammary cells may contribute to improving efficiency and quality of ruminant milk production.

The lactating mammary gland is one of the most activate in terms of synthesis, utilization, and transport of FA. The FA transport process is related to the function 
of several key proteins (Haunerland and Spener, 2004; Wang et al., 2015) such as fatty acid binding proteins (FABP). The integral membrane protein coded by CD36 is believed to physically transport the FA across the plasma membrane (Pepino et al., 2014). A growing body of data suggests that FA uptake also requires concomitant FA activation to acyl-CoA (Mashek and Coleman, 2006). The protein long-chain FA acyl-CoA synthetase (ACSL) plays a central role in the FA uptake and also is associated with lipid droplet secretion (LD; Grevengoed et al., 2014). Once activated to acyl-CoA, FA have diverse cellular fates, but the 2 predominant routes of metabolism are degradation via oxidation or incorporation into complex lipids (e.g., triacylglycerol, TAG; Bionaz and Loor, 2008a). In the lactating mammary gland, the FA are mainly incorporated into lipid droplets and secreted out of the epithelial cells. Recent data underscored the role of adipocyte differentiation-related protein (ADRP) in promoting LD formation and secretion in dairy goat mammary epithelial cells (GMEC; Shi et al., 2015b). A growing body of data supports the view that the process of FA transport is regulated by multiple transcription factors, including PPAR (Bionaz et al., 2013; Shi et al., 2014b).

Data in GMEC demonstrating that PPARD activation by the synthetic ligand GW0742 (GW) upregulated the expression of genes containing $F A B P 4$ and $A D R P$ suggested a role of PPARD in promoting FA transport (Shi et al., 2017). Furthermore, the knockdown of PPARD selectively downregulated ACSL1 and $A D R P$ mRNA abundance in GMEC, underscoring a role for PPARD in lipid transport (Shi et al., 2017). It is hypothesized that overexpression of PPARD would upregulate the mRNA expression of genes related to FA transport and lipid droplet formation in ruminant mammary cells at least in part through binding the promoter region.

To investigate the role of PPARD in regulating FA transport and lipid droplet formation in ruminant mammary cells, in this study, goat PPARD was overexpressed in GMEC. The activity of the $A D R P$ promoter was also measured to assess the effect of PPARD on $A D R P$ transcription.

\section{MATERIALS AND METHODS}

\section{Adenovirus Generation}

The PPARD (GenBank no. XM_004018768.3) cDNA was cloned from GMEC, and then subcloned into the pAdTrack-CMV plasmid vector to generate pAdTrackCMV-PPARD vector. The vector was inserted into an adenoviral vector (pAdEasy-1) to generate adenoviral plasmids in BJ5183 cells. The adenoviral plasmids linearized by PacI (New England Biolabs, Ipswich, MA) were transfected into 293A cells to generate the adenoviral Ad-PPARD. The generation and proliferation of adenovirus (Ad-PPARD) was performed as previously described (Shi et al., 2013a). The 293A cell line for adenovirus generation was cultured in basal Dulbecco's modified Eagle medium (Gibco, Waltham, MA) containing $10 \%$ fetal bovine serum. A recombinant adenovirus of green fluorescent protein (Ad-GFP) was used as a control.

\section{Cell Culture and Treatments}

The GMEC were isolated from peak lactation Xinong Saanen goats as described previously (Shi et al., 2014a). The GMEC were isolated from 3 individual goats. Details of cell culture are described elsewhere (Shi et al., 2013b, 2014a). Briefly, the collected mammary tissues were pooled together and cut into small pieces using scissors before plating on cell culture dishes. The GMEC were isolated from this mixture of tissue. To promote lactogenesis, GMEC were cultured in a lactogenic medium for $24 \mathrm{~h}$ before initial experiments. Lactogenic medium was composed of Dulbecco's modified Eagle medium/F12 (Hyclone, Beijing, China), insulin (5 mg/L, Sigma-Aldrich, St. Louis, MO), hydrocortisone $(5 \mathrm{mg} / \mathrm{L}$, Sigma-Aldrich), penicillin/streptomycin (10,000 units/L, Harbin Pharmaceutical Group, Harbin, China), prolactin (2 $\mu \mathrm{g} / \mathrm{mL}$, Sigma-Aldrich), and BSA (1 g/L, Sigma-Aldrich).

For the adenovirus infection, the GMEC cultured in 6-well plates were incubated with adenovirus medium Ad-GFP or Ad-PPARD, respectively. Treated GMEC were cultured with $1 \mu M \mathrm{GW}$ [final concentration, diluted in dimethyl sulfoxide (DMSO), Sigma-Aldrich] or control (DMSO, Sigma-Aldrich) after $24 \mathrm{~h}$ of initial culture, and then harvested at $48 \mathrm{~h}(24 \mathrm{~h}$ later $)$ for RNA extraction, cellular TAG assay, or lipid extraction. The adenovirus Ad-GFP was used as a control.

\section{Total RNA Extraction and Quantitative PCR}

Total RNA was extracted using the RNA Prep pure cell Kit (Tiangen Biotech Co. Ltd., Beijing, China) and quantitative PCR (qPCR) was performed according to the manufacturer's instructions using SYBR Green (SYBR Premix Ex Taq II, Perfect Real Time, Takara Bio Inc., Dalian, China; Shi et al., 2014b). Genes studied included PPARD, ACSL1, ADRP, CD36, FABP3, $F A B P 4$, and $S C D 1$. All the $\mathrm{qPCR}$ reactions were performed in a Bio-Rad CFX96 (Bio-Rad Laborato- 
ries Inc., Hercules, CA) sequence detector, and data were normalized to the geometric mean of ubiquitously expressed transcript $(U X T)$, mitochondrial ribosomal protein L39 (MRPL39), and ribosomal protein S9 (RPS9). The sequences of the primers for the genes are reported in Supplemental Table S1 (https://doi.org/10 .3168/jds.2017-13543).

\section{Lipid Extraction and Analysis}

Collected GMEC were washed 3 times with PBS and then scraped off the culture dish using a $2-\mathrm{mL}$ aliquot of $2.5 \%$ ( vol/vol) vitriol:methanol. Then, total lipid extraction and methylation was performed according to Shi et al. (2013a). Methylated lipid samples were analyzed using a GC-MS (Agilent Technologies, Santa Clara, CA) installed with an HP-5 column $(35 \mathrm{~m} \times$ $0.25 \mathrm{~mm}$ i.d. $\times 0.25 \mu \mathrm{m}$ film thickness) following a published procedure (Yao et al., 2016). The relative proportion of each FA was determined as the ratio of the FA peak to the total peaks in each run. The data for each FA were analyzed as a proportion of the total FA.

\section{Vector Construction}

The goat $A D R P$ promoter sequence was amplified from goat blood genomic DNA according to a previous publication (Kang et al., 2015). The PCR primers were designed to hybridize at $-1,868,-1,444,-990$, -788 , and -38 bp to generate the corresponding deletion plasmid derivatives using the common downstream primer at +516 bp (deletion primers are provided in Supplemental Table S2; https://doi.org/10.3168/jds .2017-13543). The resulting amplicon was cloned into the pGL3-Basic vector (Promega, Madison, WI) by using KpnI/BglII (Takara Bio Inc.) enzyme sites.

According to the reported PPRE (-GGGGCAGGAGGAGA-, $-1,003$ to $-990 \mathrm{bp}$ ), site-directed mutagenesis was performed to mutate the PPRE site in the ADRP promoter wild type (ADRP-wild, $-1,868$ to +516 bp) by altering 3 nucleotides within each site ("AGG" in original PPRE to "GTC"; Kang et al., 2015). The resulting amplicons were ligated to the pGL3-basic vector after digestion with $K p n \mathrm{I} / B g l \mathrm{II}$ (Takara Bio Inc.) to create a mutant construct (ADRP-mut). All the plasmids were confirmed by DNA sequencing.

\section{RNA Interference}

To knockdown the expression of PPARD (GenBank no.: XM_004018768.3), small interfering RNA (siNC, siPPARD) were designed and synthesized by Jima
Corp. (Shanghai, China). A scrambled siRNA was designed and used as a negative control. The siRNA sequence is 5'-GCUG GCCUCCAUUGUCAAUTT-3' (Shi et al., 2017).

\section{Vector Transfection}

For the luciferase assay, the GMEC cultured in 48well plates at 80 to $90 \%$ confluent were co-transfected with $290 \mathrm{ng}$ of the vector (ADRP-wild, ADRP-mut, or deletion-fragment of $A D R P$ promoter) plus $10 \mathrm{ng}$ of control vector (renilla luciferase) per well using Lipofectamine 2000 Reagent (Invitrogen, Carlsbad, CA) according to the manufacturer's protocols. For the transfection of ADRP-wild or ADRP-mut, transfected GMEC were incubated with adenovirus medium (Ad-GFP or Ad-PPARD), or incubated with siRNA (siNC or siPPARD) using Lipofectamine RNAiMAX (Invitrogen) after $12 \mathrm{~h}$ of initial culture. The transfected GMEC were treated with $1 \mu M \mathrm{GW}$ (final concentration, diluted in DMSO, Sigma-Aldrich) or control (DMSO, with the same volume as GW solution) after $36 \mathrm{~h}$ of initial culture, and then harvested at $56 \mathrm{~h}$ for luciferase activity assays.

Based on the fact that a concentration of GW of 1 to $10 \mu M$ could markedly increase the activation of PPRE in GMEC (Shi et al., 2017), this compound was chosen to assess the response of ADRP-wild to PPARD activation. After $12 \mathrm{~h}$ of initial culture, the transfected GMEC were treated with $1 \mu M, 5 \mu M \mathrm{GW}$ (final concentration, diluted in DMSO, Sigma-Aldrich), or control $(0 \mu M$, with the same volume as the GW solution) after $24 \mathrm{~h}$ of initial culture, and then harvested at $48 \mathrm{~h}$ for luciferase activity assays.

To assess the activity of deletion fragments of the $A D R P$ promoter in response to PPARD activation, after $12 \mathrm{~h}$ of initial culture, the transfected GMEC were treated with $5 \mu M \mathrm{GW}$ or DMSO after $24 \mathrm{~h}$ of initial culture, and then harvested at $48 \mathrm{~h}$ for luciferase activity assays.

\section{Luciferase Analysis}

After collection, the GMEC were washed 3 times with PBS and then incubated with passive lysis buffer (Promega) for $30 \mathrm{~min}$ at room temperature. The dual-Luciferase Reporter assay kit (Promega) was used to measure luciferase activity on a Fluoroskan Ascent apparatus (Thermo Fisher Scientific, Waltham, MA) according to the kit manufacturer's protocol (Shi et al., 2017). The relative luciferase activity was calculated as the ratio of firefly luciferase compared with renilla luciferase activity. 


\section{Cellular TAG Assay}

Total cellular TAG was extracted according to the protocol of the GPO-Trinder triglyceride assay kit (Applygen Technologies, Beijing, China). The concentration of TAG was determined according to the manufacturer's instructions on a microtiter plate reader (BioTek, Winooski, VT). The concentrations were calculated using the equation obtained from linear regression of the standard curve. The quantification of total cellular TAG was normalized to the cellular protein concentration. Protein concentration of each well was determined using a BCA protein assay (Pierce, Thermo Fisher Scientific) according to the manufacturer's instructions (Shi et al., 2017).

\section{Statistical Analysis}

Treatments were replicated at least 3 times, and results are expressed as mean \pm standard error of the mean. Data from qPCR were analyzed using the $2^{-\Delta \Delta \mathrm{Ct}}$ method (Livak and Schmittgen, 2001) and normalized to the control (Ad-GFP+DMSO). Statistical differences of mRNA expression and FA profile and activity of ADRP-wild treated with GW at different concentration were determined with a 1-way ANOVA using SPSS 19.0 (SPSS Inc./IBM Corp., Chicago, IL). Statistical analyses of each fragment of ADRP promoter activation were determined with a $t$-test (paired and 2-tailed; SPSS Inc./IBM Corp.). The activity of ADRP-wild or ADRP-mut were expressed within knockdown (siPPARD) or overexpression (Ad-PPARD) of PPARD, or control (Ad-GFP or siNC). This approach allowed us to assess directly the effect of different treatments for a given factor. Statistical differences were determined with a 1-way ANOVA using SPSS 19.0. Differences were considered statistically significant when $P<0.05$.

\section{RESULTS}

\section{Overexpression of PPARD Increased the mRNA Abundance of Genes Related to Lipid Transport}

To assess the effect of PPARD on the expression of genes related to lipid transport, PPARD was overexpressed in the GMEC using adenovirus (Ad-PPARD), with Ad-GFP as the control. As shown in Figure 1A, compared with the control (Ad-GFP+DMSO), incubation with the ligand (GW) had no effect on the expression of PPARD in the cells infected with Ad-GFP. Overexpression of PPARD without (Ad-PPARD+DMSO) or with (Ad-PPARD+GW) GW markedly upregulated the expression of PPARD compared with the control
$($ Ad-GFP+DMSO $)$ and GW (Ad-PPARD+DMSO) treatments (Figure 1A). However, PPARD overexpression alone did not $(P>0.05)$ increase mRNA expression of $S C D 1$ and $C D 36$ compared with the control (Ad-GFP+DMSO). The addition of GW to cultures overexpressing PPARD (Ad-PPARD+GW) upregulated the expression of these 2 genes by $\sim 3$ - and $\sim 17$-fold, respectively, relative to the control (AdGFP+DMSO; Figure 1B). Compared with the control (Ad-GFP+DMSO), the cells incubated with GW had a higher CD36 mRNA expression, but not SCD1 mRNA.

Compared with the control (Ad-GFP+DMSO), the addition of GW to cultures did not affect the mRNA abundance of FABP3 $(P>0.05)$ but increased the expression of FABP4 $(P<0.05$; Figure $1 \mathrm{C})$. Compared with the control (Ad-GFP+DMSO), the mRNA expression of FABP3 and FABP 4 was upregulated $(P$ $<0.05)$ when the transfected cells (Ad-PPARD+GW) were incubated with GW. Relative to the control (Ad$\mathrm{GFP}+\mathrm{DMSO}$ ), the mRNA of FABP3 and FABP4 increased by $\sim 1.8$ - and 2-fold, respectively. Compared with the control (Ad-GFP+DMSO), the cells overexpressing PPARD (Ad-PPARD+DMSO) resulted in a decrease of $F A B P 3$ mRNA but had no effect on FABP 4 mRNA expression.

Compared with the control (Ad-GFP+DMSO), genes encoding proteins associated with $\mathrm{FA}$ activation (ACSL1) and lipid droplet secretion $(A D R P)$ were upregulated $(P<0.05)$ in cultures incubated with $\mathrm{GW}$ (Figure 1D). Addition of GW to cells overexpressing PPARD enhanced the mRNA abundance of these 2 genes compared with the control (Ad-GFP+GW). Relative to the control (Ad-GFP+DMSO), the mRNA of ACSL1 and ADRP in cells overexpressing PPARD and incubated with GW increased by $\sim 3.8$ - and $\sim 35$-fold, respectively. Overexpressing PPARD alone, however, did not increase expression of these genes compared with the control (Ad-GFP+DMSO).

\section{Overexpression of PPARD Altered the Cellular Fatty Acid Profile}

The FA profile in GMEC was measured after PPARD overexpression. As shown in Table 1, compared with the control (Ad-GFP+DMSO), treatment with GW had no effect $(P>0.05)$ on the concentration of C14:0, C16:0, C16:1, C18:0, C18:1-trans, and C18:1-cis. However, the ratio of $\mathrm{C} 16: 1(P<0.05)$ and $\mathrm{C} 18$ :1-trans $(P<0.05)$ in cells overexpressing $P P A R D$ and incubated with GW increased significantly at the expense of C16:0 $(P<$ $0.05)$ and $\mathrm{C} 18: 0(P<0.05)$. Compared with the control (Ad-GFP+DMSO), overexpression of PPARD had no effect on the ratio of $\mathrm{C} 14: 0$ and $\mathrm{C} 18: 1-$ cis. 

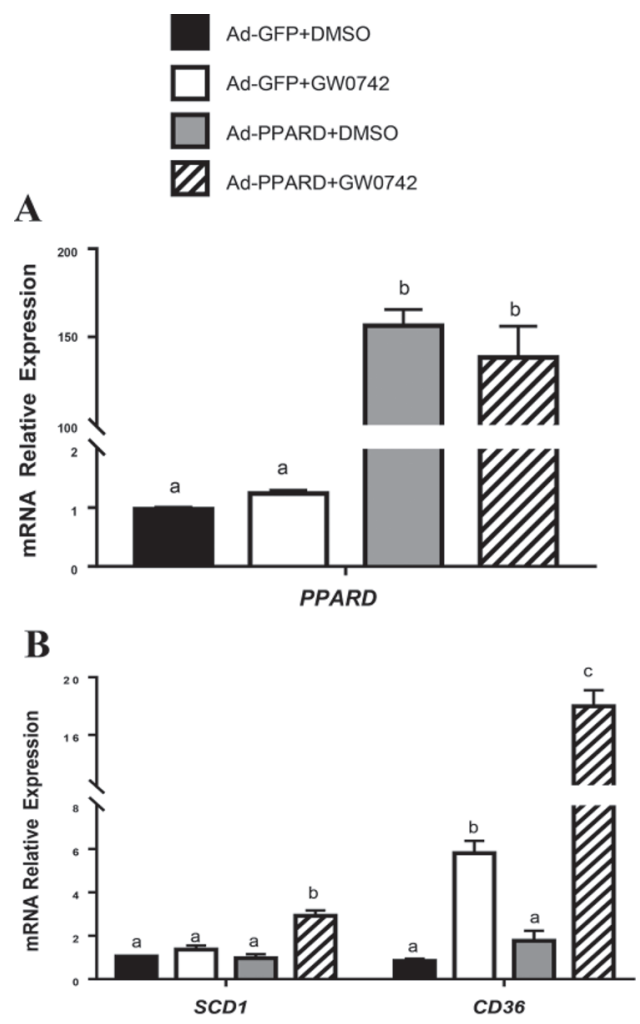

C

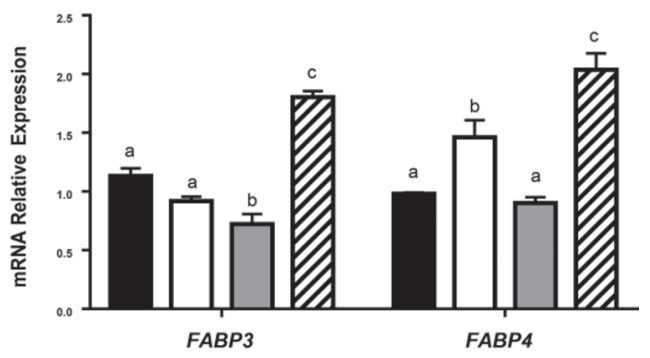

D

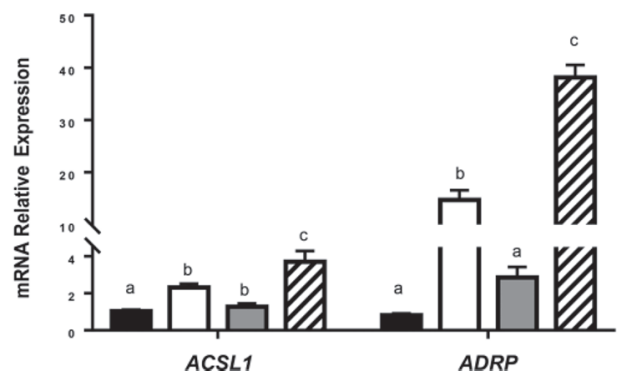

Figure 1. Overexpression of peroxisome proliferator activated receptor delta $(P P A R D)$ affects the expression of genes related to lipid transport in goat mammary epithelial cells (GMEC). After a 24-h infection with Ad-GFP (control; adenovirus containing green fluorescent protein) or Ad-PPARD (adenovirus containing PPARD), the GMEC were incubated with dimethyl sulfoxide (DMSO) or the PPARD ligand GW0742 for $24 \mathrm{~h}$. The total RNA was extracted to assess the gene expression changes. Panel A: the mRNA expression of PPARD. Panel B: the mRNA expression of $S C D 1$ and $C D 36$. Panel C: the mRNA expression of FABP 3 and FABP4. Panel D: the mRNA expression of ACSL1 and $A D R P$. Data are means \pm SEM from 3 replicates. Different letters denote significant $(P<0.05)$ differences in expression.

\section{Overexpression of PPARD Reduced the Cellular TAG Concentration}

Compared with the control (Ad-GFP+DMSO), incubation with GW did not alter the cellular TAG profile in GMEC (Figure 2). Compared with the control (Ad$\mathrm{GFP}+\mathrm{DMSO}$ ), the overexpression of PPARD in GMEC incubated with $(P<0.05)$ or without $(P<0.05) \mathrm{GW}$ reduced the total cellular TAG. However, no significant change occurred in TAG in cells overexpressing PPARD compared with the control (Ad-GFP+GW; Figure 2).

\section{Activation of PPARD by GW Enhanced the Promoter Activity of ADRP}

To investigate the role of PPARD in promoting lipid droplet formation, the activity of $A D R P$ promoter fragments was measured. As shown in Figure 3A, GW increased the activity of the ADRP promoter in a dosedependent manner. The cells incubated with GW at 5 $\mu M$ had the highest relative luciferase level. Treatment with GW at $1 \mu M$ also increased the relative luciferase level compared with the control $(\mathrm{GW}$ at $0 \mu M)$.

A series of 5 c-flanking deletion constructs $(-1,868 /+516,-1,444 /+516,-990 /+516,-788 /+516$, and $-38 /+516 \mathrm{bp}$ ) of the ADRP promoter were generated and transfected into GMEC. Results in Figure $4 \mathrm{~B}$ revealed no reduction in the activity of the $A D R P$ promoter due to deletion fragments of $-1,868 /+516$, $-1,444 /+516,-990 /+516$, and $-788 /+516 \mathrm{bp}$, whereas a dramatic decrease in $A D R P$ promoter activity was observed when deleting up to $-38 /+516$ bp (Figure 3B). Compared with each control (cells incubated with DMSO), the PPARD agonist GW significantly increased the activation of $-1,868 /+516$ bp $(P<0.05)$ and $-1,444 /+516$ bp $(P<0.05)$ fragments, but failed to do so with $-990 /+516,-788 /+516$, and $-38 /+516$ bp fragments $(P>0.05)$.

\section{PPARD Promoted the Activity of ADRP Through Binding to the PPRE}

To further investigate the mechanism whereby PPARD enhances ADRP promoter activation, PPARD was knocked down using siRNA (siPPARD) or overexpressed in GMEC that were transfected with the $A D R P$ promoter. In the cells incubated with siNC, treatment with $\mathrm{GW}$ increased $(P<0.05)$ the activity of ADRP-wild compared with the control (cells treated with DMSO). However, knockdown of PPARD impaired the induction of GW on ADRP-wild activity $(P>0.05$; Figure 4A). In the cells incubated with AdGFP, GW increased $(P<0.05)$ the activity of ADRPwild compared with the control (Ad-GFP+DMSO). In 


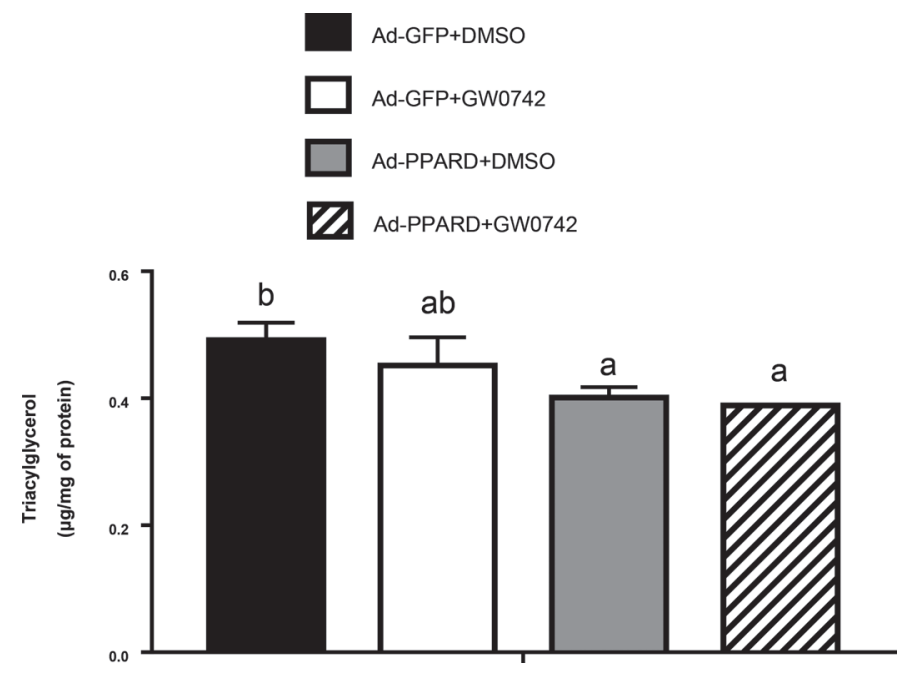

Figure 2. Changes in total cellular triacylglycerol in goat mammary epithelial cells (GMEC) in response to overexpression of peroxisome proliferator-activated receptor delta (PPARD; Ad-PPARD+DMSO), incubation with the PPARD ligand GW0742 (Ad-GFP+GW0742) or without GW0742 (Ad-GFP+DMSO), or overexpression of PPARD plus GW0742 (Ad-PPARD+GW0742). Data are means \pm SEM from 3 individual cultures. Different letters denote significant $(P<0.05)$ differences. DMSO = dimethyl sulfoxide

contrast, in the cells transfected with Ad-PPARD, GW significantly upregulated the activity of ADRP-wild compared with the control (Ad-PPARD+DMSO; Figure 4B). In cells transfected with ADRP-mut, addition of GW had no effect on the relative luciferase activity regardless of the overexpression of PPARD $(P>0.05$; Figure 4C).

\section{DISCUSSION}

In rodents, the role of PPARD is primarily associated with FA oxidation (Schuler et al., 2016). Recent data in GMEC support a role of PPARD in facilitating lipid secretion and catabolism of FA (Shi et al., 2017). However, the exact mechanism for the PPARD effect in mammary cells has not been fully resolved. In the pres- ent study, the role of PPARD on FA transport was assessed using a combination of activation with GW and alteration of PPARD expression. The novel findings underscore an important role of PPARD in regulating FA transport directly via binding to the promoter of downstream genes (e.g., ADRP).

The gene encoding the FA translocase CD36 is a target of PPAR in rodents (Zhou et al., 2008) and was markedly upregulated by overexpression of $P P A R G$ in GMEC (Shi et al., 2014b). The finding that the $C D 36$ expression was increased in the cells overexpressing PPARD following incubation with GW is highly suggestive that CD36 is a target of PPARD in GMEC.

Fatty acid binding proteins are thought to be the main intracellular FA transporters in ruminant cells (Bionaz and Loor, 2008a). The increase of FABP4 in cultures incubated with both GW or overexpressing PPARD plus GW is consistent with the fact that $F A B P 4$ is a target of PPAR (Shin et al., 2009). The lack of effect of GW on the expression of FABP3 agrees with previous data in GMEC (Shi et al., 2017), and suggests it is not a target of PPARD. However, the fact that overexpression of PPARD plus GW increased the mRNA level of $F A B P 3$ seems to suggest an alternate mechanisms whereby activation of PPARD may induce activation of another transcription regulator controlling the expression of FABP3. This idea is supported by the fact that $F A B P 3$ has the highest expression level among $F A B P$ in the lactating ruminant mammary gland (Bionaz and Loor, 2008b).

Esterification with CoA catalyzed by ACSL is essential for the participation of long-chain FA in metabolic pathways or cellular transport. The high expression of ACSL1 suggests an important role for this isotype in the lactating dairy goat mammary gland (Shi et al., 2015a). In this study, the significant increase of ACSL1 mRNA after overexpression of PPARD is consistent with the fact that knockdown of PPARD decreased ACSL1 expression (Shi et al., 2017). Because esterification with $\mathrm{CoA}$ enhances FA transport (Mashek and

Table 1. Effect of overexpression of peroxisome proliferator-activated receptor delta $(P P A R D)$ on the fatty acid composition (\%; mean \pm SEM) in goat mammary epithelial cells ${ }^{1}$

\begin{tabular}{lccc}
\hline Fatty acid & Ad-GFP+DMSO & Ad-GFP+GW0742 & Ad-PPARD+GW0742 \\
\hline C14:0 & $0.89 \pm 0.17$ & $1.06 \pm 0.1$ & $0.97 \pm 0.07$ \\
C16:1 & $1.06 \pm 0.01^{\mathrm{a}}$ & $1.17 \pm 0.04^{\mathrm{ab}}$ & $1.45 \pm 0.34^{\mathrm{b}}$ \\
C16:0 & $22.18 \pm 0.44^{\mathrm{b}}$ & $22.87 \pm 1.26^{\mathrm{ab}}$ & $21.64 \pm 0.19^{\mathrm{a}}$ \\
C18:1-trans & $26.13 \pm 0.55^{\mathrm{a}}$ & $27.22 \pm 1.46^{\mathrm{ab}}$ & $27.71 \pm 0.2^{\mathrm{b}}$ \\
C18: 1 - cis & $4.88 \pm 0.17$ & $4.8 \pm 0.18$ & $5.08 \pm 0.22$ \\
C18:0 & $13.52 \pm 0.4^{\mathrm{b}}$ & $13.53 \pm 1.10^{\mathrm{ab}}$ & $12.27 \pm 0.00^{\mathrm{a}}$ \\
\hline
\end{tabular}

$\overline{a, b}$ Values within a row with different superscripts differ significantly $(P<0.05)$.

${ }^{1} \mathrm{Ad}-\mathrm{GFP}=$ recombinant adenovirus of green fluorescent protein as control; Ad-PPARD = recombinant adenovirus containing peroxisome proliferator-activated receptor delta. DMSO = dimethyl sulfoxide; GW0742 $=$ synthetic ligand. 
Coleman, 2006), an upregulation of ACSL1 in GMEC was hypothesized to enhance FA transport and efficiency of FA metabolism for TAG synthesis (Shi et al., 2017). This idea is supported by observations in dairy cow mammary epithelial cells demonstrating that ACSL1 activation enhanced TAG synthesis (Lian et al., 2016), and in mouse liver where the loss of ACSL1 decreased TAG synthesis (Li et al., 2009). These data suggest PPARD may promote the synthesis of TAG via controlling the activation of ACSL1.
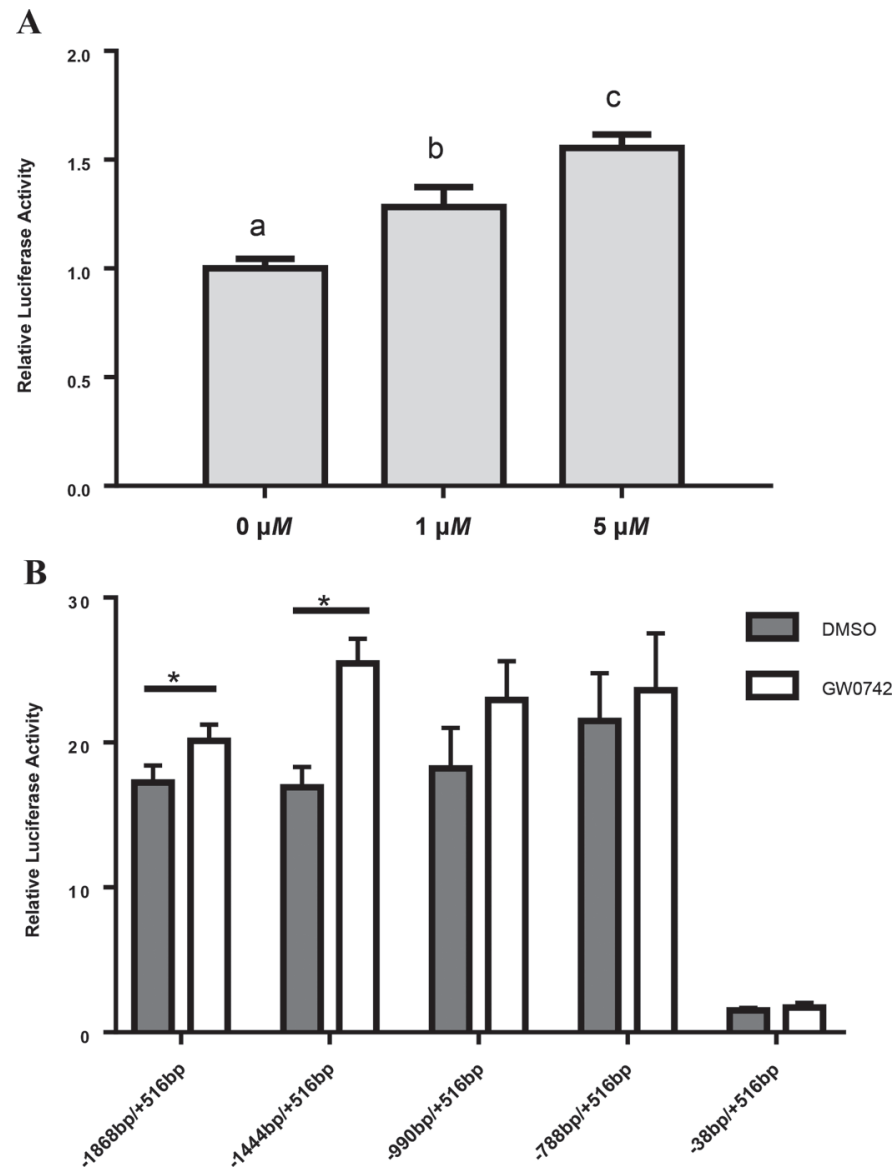

Figure 3. The compound GW0742 (GW) activated the peroxisome proliferator-activated receptor delta $(P P A R D)$ promoter activity in goat mammary epithelial cells (GMEC). Panel A: Cells were co-transfected with adipocyte differentiation-related protein $(A D R P)$ promoter and Renilla luciferase vectors. After transfection for $24 \mathrm{~h}$, cells were treated with GW at 0,1 , and $5 \mu M$ for $24 \mathrm{~h}$, respectively. The relative luciferase activity is expressed relative to the control $(0$ $\mu M)$ and normalized with renilla luciferase activity, respectively. Panel B: After $24 \mathrm{~h}$ co-transfection with each 5 '-flanking deletion construct $(-1,868 /+516 \mathrm{bp},-1,444 /+516 \mathrm{bp},-990 /+516 \mathrm{bp},-788 /+516 \mathrm{bp}$, or $-38 /+516 \mathrm{bp}$ ) and pRL-TK vectors, cells were treated with GW at $5 \mu M$ for $24 \mathrm{~h}$. The relative luciferase activity of each fragment is expressed relative to the control (dimethyl sulfoxide; DMSO) and normalized with renilla luciferase activity. The data represent mean \pm SEM from replicates. Different letters denote significant $(P<0.05)$ differences in relative luciferase activity in panel A. In panel B, asterisks denote significant $(P<0.05)$ differences.
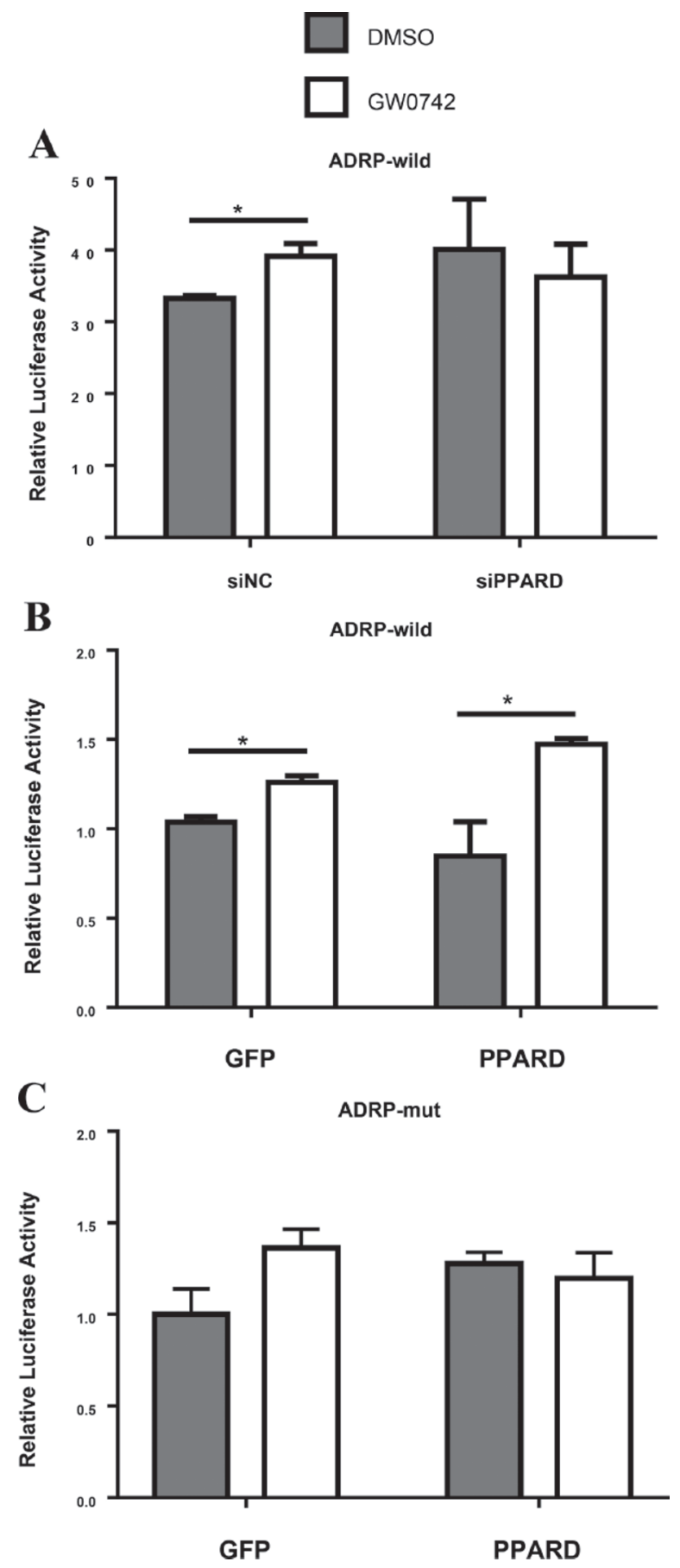

Figure 4. Overexpression of peroxisome proliferator-activated receptor delta $(P P A R D)$ or knockdown of $P P A R D$ altered the activity of adipocyte differentiation-related protein $(A D R P)$ promoter in goat mammary epithelial cells (GMEC) containing a wild type construct (ADRP-wild) or a mutation of the PPARG response element (ADRP-mut) and incubated with the PPARD agonist GW0742 (GW) or dimethyl sulfoxide (DMSO). A vector containing renilla luciferase was used as control and co-transfected with each ADRP promoter in GMEC. Panels A and B: relative luciferase activation of ADRP-wild; panel C: relative luciferase activation of ADRP-mut. Values are means \pm SEM from 3 individual cultures. Asterisks denote significant $(P<$ 0.05 ) differences. $\mathrm{GFP}=$ green fluorescent protein; $\operatorname{siNC}=$ small $\mathrm{RNA}$ as negative control; siPPARD = small RNA interfering PPARD. 
The finding that overexpression of $P P A R D$ resulted in a decrease of TAG concentration is noteworthy. It is possible that the mechanism resulting in decrease of TAG is mainly caused by changes in $\beta$-oxidation. Previous work with nonruminants has clearly identified PPARD as an activator of genes required for FA oxidation (Wang et al., 2003; Gan et al., 2011). Our recent work also provided evidence in GMEC that activation of PPARD upregulated genes related to $\beta$-oxidation of FA (Shi et al., 2017). The decrease of TAG is consistent with the finding in mice that activation of PPARD by chemical agonists causes lipid depletion (Wang et al., 2003). A limitation of the present study is that the secreted TAG in the culture medium could not be assessed due to the extremely low concentration in the serum-free in vitro culture (Li et al., 2014). Further in vivo and vitro experiments will have to be conducted to verify the mechanism of TAG metabolism in response to PPARD activation. Despite this limitation, the data still support an important role for PPARD in lipid homeostasis in the lactating ruminant mammary gland.

Consistent with the mRNA expression of ADRP induced by GW, GW treatment increased the activity of the ADRP promoter in a dose-dependent manner. The finding in cultures treated with GW that knockdown of PPARD impaired the activity of ADRP-wild and that overexpression of $P P A R D$ enhanced the activity of ADRP-wild strongly suggest that $A D R P$ is a target of PPARD. By transiently transfecting the ADRP 5 '-flanking truncated luciferase reporter vector into GMEC, we identified a core region at $-1,444$ to -990 $\mathrm{bp}$ that is responsive to PPARD activation (Figure $4 \mathrm{~B})$. The core region is consistent with the previous finding that the PPRE spanning at $-1,003$ to $-990 \mathrm{bp}$ on the ADRP promoter was responsive to the activation of PPARG (Kang et al., 2015). Furthermore, the data that a PPRE mutation impaired the activity of $A D R P$ promoter induced by $\mathrm{GW}$ and overexpression of PPARD indicate that PPARD regulates ADRP expression by directly binding to its promoter through a similar regulatory mechanism as PPARG (Kang et al., 2015).

The upregulation of $S C D 1$ in the cells overexpressing PPARD and cultured with GW is consistent with observations in mouse liver, where adenovirus-mediated restricted PPARD activation in the liver and increased the mRNA level of SCD1 (Liu et al., 2011). The increase of MUFA concentrations may have been due to the upregulation of $S C D 1$ after treatment with AdPPARD and GW. Overexpression of PPARD plus GW increased the ratio of $\mathrm{C} 16: 1$ and $\mathrm{C} 18: 1$ at the expense of C16:0 and C18:0 and led to a similar pattern when PPARG was overexpressed and incubated with rosiglitazone in GMEC (Shi et al., 2013a). This suggests that both PPARD and PPARG may have overlapping functions in regulating FA desaturation. The similar change in FA profiles elicited by PPARG and PPARD also suggest these 2 isotypes may share a functional PPRE in the SCD1 promoter (Yao et al., 2017). However, the fact that GW did not induce $S C D 1$ expression strongly suggests a different PPRE binding model in the promoter for these 2 isotypes (at least for $S C D 1$ ). This phenomenon may partly result from differences in the co-activator proteins that work with PPAR (Liu et al., 2011).

Previous data indicated that $A D R P, S C D 1$, and FABP 4 are targets of PPARG (Bionaz et al., 2013); thus, the induction of these genes by $P P A R D$ suggests a functional overlap between PPARD and PPARG. This idea agrees with the fact in rodents that PPAR are activated by overlapping groups of FA derivatives and share many target genes (Poulsen et al., 2012). However, the different regulatory mechanism on $S C D 1$ also suggest a distinct function for each isotype. The distinct function for PPARD is partly related to the different biochemical properties responsible for the integration of the PPARD subtypes into transcriptional complexes and signaling networks (Wang, 2010; Poulsen et al., 2012).

Collectively, the data reveal a novel role of PPARD in regulating lipid transport and promoting LD formation in GMEC. This role could be helpful for manipulating milk lipid synthesis in the lactating ruminant mammary gland. Our data also indicate that the mechanism of action of PPARD is through regulating downstream gene expression via binding to the promoter (at least on $A D R P)$.

\section{CONCLUSIONS}

The combination of gene overexpression and promoter analysis underscore a novel role for PPARD in lipid homeostasis via promoting lipid transport. This function involves the cellular FA trafficking and LD formation. There appears to be a functional overlap in GMEC among the PPAR family. More controlled molecular experiments will have to be performed to address questions about the functional overlap among PPAR. Together, results reveal a crucial role for PPARD in the regulation of milk fat metabolism.

\section{ACKNOWLEDGMENTS}

This research was jointly supported by a National Natural Science Foundation of China (31702090, Beijing, China), Open Fund of Zhejiang Provincial Top Key Discipline of Biology (Hangzhou, China), and a Special Fund for Agro-Scientific Research in the Public 
Interest of Zhejiang Province (2016C32061, Hangzhou, China).

\section{REFERENCES}

Bionaz, M., S. Chen, M. J. Khan, and J. J. Loor. 2013. Functional role of PPARs in ruminants: Potential targets for fine-tuning metabolism during growth and lactation. PPAR Res. 2013:28.

Bionaz, M., and J. Loor. 2008a. Gene networks driving bovine milk fat synthesis during the lactation cycle. BMC Genomics 9:366.

Bionaz, M., and J. J. Loor. 2008b. ACSL1, AGPAT6, FABP3, LPIN1, and SLC27A6 are the most abundant isoforms in bovine mammary tissue and their expression is affected by stage of lactation. J. Nutr. 138:1019-1024.

Gan, Z., E. M. Burkart-Hartman, D.-H. Han, B. Finck, T. C. Leone, E. Y. Smith, J. E. Ayala, J. Holloszy, and D. P. Kelly. 2011. The nuclear receptor PPAR $\beta / \delta$ programs muscle glucose metabolism in cooperation with AMPK and MEF2. Genes Dev. 25:2619-2630.

Giordano Attianese, G. M. P., and B. Desvergne. 2015. Integrative and systemic approaches for evaluating PPAR $\beta / \delta$ (PPARD) function. Nucl. Recept. Signal. 13:e001.

Grevengoed, T. J., E. L. Klett, and R. A. Coleman. 2014. Acyl-CoA metabolism and partitioning. Annu. Rev. Nutr. 34:1-30.

Harvatine, K. J., Y. R. Boisclair, and D. E. Bauman. 2009. Recent advances in the regulation of milk fat synthesis. Animal 3:40-54.

Haunerland, N. H., and F. Spener. 2004. Fatty acid-binding proteinsInsights from genetic manipulations. Prog. Lipid Res. 43:328-349.

Kang, Y., S. Hengbo, L. Jun, L. Jun, Z. Wangsheng, T. Huibin, and S. Huaiping. 2015. PPARG modulated lipid accumulation in dairy GMEC via regulation of ADRP gene. J. Cell. Biochem. 116:192201.

Li, L. O., J. M. Ellis, H. A. Paich, S. Wang, N. Gong, G. Altshuller, R. J. Thresher, T. R. Koves, S. M. Watkins, D. M. Muoio, G. W. Cline, G. I. Shulman, and R. A. Coleman. 2009. Liver-specific loss of long chain acyl-CoA synthetase-1 decreases triacylglycerol synthesis and beta-oxidation and alters phospholipid fatty acid composition. J. Biol. Chem. 284:27816-27826.

Li, N., F. Zhao, C. Wei, M. Liang, N. Zhang, C. Wang, Q. Z. Li, and X. J. Gao. 2014. Function of SREBP1 in the milk fat synthesis of dairy cow mammary epithelial cells. Int. J. Mol. Sci. 15:16998-17013

Lian, S., J. R. Guo, X. M. Nan, L. Ma, J. J. Loor, and D. P. Bu. 2016. MicroRNA Bta-miR-181a regulates the biosynthesis of bovine milk fat by targeting ACSL1. J. Dairy Sci. 99:3916-3924.

Liu, S., B. Hatano, M. Zhao, C. C. Yen, K. Kang, S. M. Reilly, M. R. Gangl, C. Gorgun, J. A. Balschi, J. M. Ntambi, and C. H. Lee. 2011. Role of peroxisome proliferator-activated receptor \{delta\}/ \{beta\} in hepatic metabolic regulation. J. Biol. Chem. 286:12371247.

Livak, K. J., and T. D. Schmittgen. 2001. Analysis of relative gene expression data using real-time quantitative PCR and the $2-\Delta \Delta \mathrm{CT}$ method. Methods 25:402-408.

Mashek, D. G., and R. A. Coleman. 2006. Cellular fatty acid uptake: The contribution of metabolism. Curr. Opin. Lipidol. 17:274-278.

Oppi-Williams, C., J. K. Suagee, and B. A. Corl. 2013. Regulation of lipid synthesis by liver $\mathrm{X}$ receptor alpha and sterol regulatory element-binding protein 1 in mammary epithelial cells. J. Dairy Sci. 96:112-121.

Pepino, M. Y., O. Kuda, D. Samovski, and N. A. Abumrad. 2014. Structure-function of CD36 and importance of fatty acid signal transduction in fat metabolism. Annu. Rev. Nutr. 34:281-303.
Poulsen, L. C., M. Siersbæk, and S. Mandrup. 2012. PPARs: Fatty acid sensors controlling metabolism. Semin. Cell Dev. Biol. 23:631-639.

Schuler, M., F. Ali, C. Chambon, D. Duteil, J.-M. Bornert, A. Tardivel, B. Desvergne, W. Wahli, P. Chambon, and D. Metzger. 2016. PGC1A expression is controlled in skeletal muscles by PPARD, whose ablation results in fiber-type switching, obesity, and type 2 diabetes. Cell Metab. 4:407-414.

Shi, H., H. Shi, J. Luo, W. Wang, A. B. Haile, H. Xu, and J. Li. 2014a. Establishment and characterization of a dairy goat mammary epithelial cell line with human telomerase (hT-MECs). Anim. Sci. J. $85: 735-743$.

Shi, H., J. Zhu, J. Luo, W. Cao, H. Shi, D. Yao, J. Li, Y. Sun, H. $\mathrm{Xu}, \mathrm{K}$. Yu, and J. Loor. 2015a. Genes regulating lipid and protein metabolism are highly expressed in mammary gland of lactating dairy goats. Funct. Integr. Genomics 15:309-321.

Shi, H. B., J. Luo, D. W. Yao, J. J. Zhu, H. F. Xu, H. P. Shi, and J. J. Loor. 2013a. Peroxisome proliferator-activated receptor-Y stimulates the synthesis of monounsaturated fatty acids in dairy goat mammary epithelial cells via the control of stearoyl-coenzyme A desaturase. J. Dairy Sci. 96:7844-7853.

Shi, H. B., J. Luo, J. J. Zhu, J. Li, Y. T. Sun, X. Z. Lin, L. P. Zhang, D. W. Yao, and H. P. Shi. 2013b. PPAR $\gamma$ regulates genes involved in triacylglycerol synthesis and secretion in mammary gland epithelial cells of dairy goats. PPAR Res. 2013:310948.

Shi, H. B., K. Yu, J. Luo, J. Li, H. B. Tian, J. J. Zhu, Y. T. Sun, D. W. Yao, H. F. Xu, H. P. Shi, and J. J. Loor. 2015b. Adipocyte differentiation-related protein promotes lipid accumulation in goat mammary epithelial cells. J. Dairy Sci. 98:6954-6964.

Shi, H. B., C. H. Zhang, W. Zhao, J. Luo, and J. J. Loor. 2017. Peroxisome proliferator-activated receptor delta facilitates lipid secretion and catabolism of fatty acids in dairy goat mammary epithelial cells. J. Dairy Sci. 100:797-806.

Shi, H. B., W. S. Zhao, J. Luo, D. W. Yao, Y. T. Sun, J. Li, H. P. Shi, and J. J. Loor. 2014b. Peroxisome proliferator-activated receptor $\gamma 1$ and $\gamma 2$ isoforms alter lipogenic gene networks in goat mammary epithelial cells to different extents. J. Dairy Sci. 97:5437-5447.

Shin, J., B. Li, M. E. Davis, Y. Suh, and K. Lee. 2009. Comparative analysis of fatty acid-binding protein 4 promoters: Conservation of peroxisome proliferator-activated receptor binding sites. J. Anim. Sci. 87:3923-3934.

Wang, G., H. L. Bonkovsky, A. de Lemos, and F. J. Burczynski. 2015. Recent insights into the biological functions of liver fatty acid binding protein 1. J. Lipid Res. 56:2238-2247.

Wang, Y. X. 2010. PPARs: Diverse regulators in energy metabolism and metabolic diseases. Cell Res. 20:124-137.

Wang, Y. X., C. H. Lee, S. Tiep, R. T. Yu, J. Ham, H. Kang, and R. M. Evans. 2003. Peroxisome-proliferator-activated receptor $\delta$ activates fat metabolism to prevent obesity. Cell 113:159-170.

Yao, D., J. Luo, Q. He, H. Shi, J. Li, H. Wang, H. Xu, Z. Chen, Y. Yi, and J. J. Loor. 2017. SCD1 alters long-chain fatty acid (LCFA) composition and its expression is directly regulated by SREBP-1 and PPARgamma 1 in dairy goat mammary cells. J. Cell. Physiol. 232:635-649.

Yao, D. W., J. Luo, Q. Y. He, M. Wu, H. B. Shi, H. Wang, M. Wang, H. F. Xu, and J. J. Loor. 2016. Thyroid hormone responsive (THRSP) promotes the synthesis of medium-chain fatty acids in goat mammary epithelial cells. J. Dairy Sci. 99:3124-3133.

Zhou, J., M. Febbraio, T. Wada, Y. Zhai, R. Kuruba, J. He, J. H. Lee, S. Khadem, S. Ren, S. Li, R. L. Silverstein, and W. Xie. 2008. Hepatic fatty acid transporter Cd36 is a common target of LXR, PXR, and PPAR $\gamma$ in promoting steatosis. Gastroenterology 134:556-567. 